\title{
SYNTHESIS, IN VITRO ANTIOXIDANT AND ANTIMICROBIAL EVALUATION OF 3-HYDROXY CHROMONE DERIVATIVES
}

\author{
PALLAVI KAMBLE*, SAILESH WADHER \\ Department of School of Pharmacy, Swami Ramanand Teerth Marathwada University, Nanded, Maharashtra, India. \\ Email: pallavimpharma@rediffmail.com
}

Received: 09 October 2017, Revised and Accepted: 07 December 2017

\section{ABSTRACT}

Objective: The objective of the present study was to synthesize a series of 3-hydroxychromone derivatives and to evaluate its in vitro antioxidant and antimicrobial activities.

Methods: 3-hydroxy chromones were synthesized using an algar flynn oyamada method which includes oxidative cyclization of 2-hydroxy chalcones in basic solution by hydrogen peroxide. 2-hydroxy chalcones were synthesized by Claisen-Schmidt condensation of substituted 2-hydroxy acetophenones with substituted aromatic aldehydes using polyethylene glycol-400 as a recyclable solvent. The synthesized compounds were evaluated for in vitro antioxidant activity by 1,1-diphenyl-2-picrylhydrazyl radical scavenging assay. In addition, these compounds were also screened for in vitro antibacterial and antifungal activity by agar cup method and Poison plate method, respectively.

Results: The structures of the synthesized compounds were characterized by infrared, ${ }^{1} \mathrm{H}$ nuclear magnetic resonance and mass spectroscopy. The antioxidant activity data revealed that all the synthesized derivatives exhibited good activity due to the presence of phenolic hydroxyl group, 4-oxo group and 2,3-double bond. Further, the activity increased with the introduction of a more phenolic hydroxyl group and adjacent methoxy group in the structure. The antimicrobial activity data showed that the compounds possess better antibacterial and antifungal activity which is attributed to the presence of phenolic hydroxyl group and 4-oxo group in the structure.

Conclusions: The use of inexpensive, eco-friendly and readily available reagents, easy work-up and high purity of products makes the procedure a convenient and robust method for the synthesis of title compounds. The presence of phenolic hydroxyl group, 4-oxo group, and 2,3-double bond in the structure is responsible for their good antioxidant and antimicrobial activities.

Keywords: Chromone, Chalcone, Claisen-Schmidt condensation, Algar Flynn Oyamada method, Antioxidant, Antibacterial, Antifungal.

(c) 2018 The Authors. Published by Innovare Academic Sciences Pvt Ltd. This is an open access article under the CC BY license (http://creativecommons. org/licenses/by/4. 0/) DOI: http://dx.doi.org/10.22159/ajpcr.2018.v11i3.22984

\section{INTRODUCTION}

Chromones are a group of naturally occurring compounds that are ubiquitous in nature, especially in plants. The word chromone is derived from the Greek word chroma, meaning "color," which indicates that many chromone derivatives can exhibit a diversity of colors [1].

Chromones are oxygen-containing heterocyclic compounds with a benzoannelated $\gamma$-pyrone ring being chromone (4H-chromen-4-one, 4H-1-benzopyran-4-one) the parent compound (Fig. 1). 3-hydroxy chromone is the class of flavonoids structurally related to flavonols (Fig. 1) [2].

Chromones are used as scaffolds for the development of bioactive compounds. These frameworks are naturally occurring derivatives containing anoxa-pyran ring [3]. The most frequently found chromonebased natural products are the 2-arylsubstituted chromones (flavonoids) carrying hydroxy and/or methoxy groups on the aromatic rings [4]. The substitution pattern of the chromone scaffolds determines their different biological effects. Known effects of these types of compounds are antioxidant [5], antiviral [6], antibacterial [7], antifungal, antiinflammatory [8], antiobesity [9], immunomodulatoty [10], and kinase inhibition [11]. Hence, chromones can be considered privileged structures, defined as "a single molecular framework able to provide ligands for diverse receptors" [12].

Prompted by all these observations, we report herein the synthesis, in vitro antioxidant and antimicrobial activities of 3-hydroxy chromone derivatives.

\section{EXPERIMENTAL}

Aldehydes and acetophenones were procured from Sigma-Aldrich and SD fine chemicals. All other chemicals are of AR grade. Melting points were determined in open capillaries on a Metal Toledo digital melting point apparatus and are uncorrected. The purity of the compounds was checked by thin-layer chromatography (TLC) using TLC Silica gel $60 \mathrm{~F}_{254}$ aluminum sheets procured from Merck and spots were detected in ultra violet fluorescence analysis cabinet. The infrared (IR) spectra were recorded using potassium bromide (KBr) pellets on Shimadzu IR Affinity-1 Fourier transform IR spectrophotometer $\left(\mathrm{cm}^{-1}\right)$. ${ }^{1} \mathrm{H}$ nuclear magnetic resonance (NMR) spectra were recorded on Bruker AVANCE III $500 \mathrm{MHz}$ NMR spectrometer using tetramethylsilane as internal standard (chemical shifts in $\delta \mathrm{ppm}$ ) and mass spectra recorded were on JEOL GC MATE II GC-MS system.

General procedure for synthesis of 2-hydroxy chalcone derivatives (Fig. 2)

An equimolar mixture of substituted acetophenone (1 mmol), aromatic aldehyde $(1 \mathrm{mmol})$ and potassium hydroxide $(2 \mathrm{mmol})$ was stirred in polyethylene glycol- 400 (PEG-400) $(15 \mathrm{ml})$ at $40^{\circ} \mathrm{C}$ for $1 \mathrm{~h}$. After completion of the reaction (monitored by TLC), the crude mixture was worked up in ice-cold water $(100 \mathrm{ml})$. The separated product was filtered, washed with water and recrystallized with suitable solvent. The filtrate was evaporated to remove water, leaving PEG behind. The same PEG was utilized to synthesize further chalcones [13] 


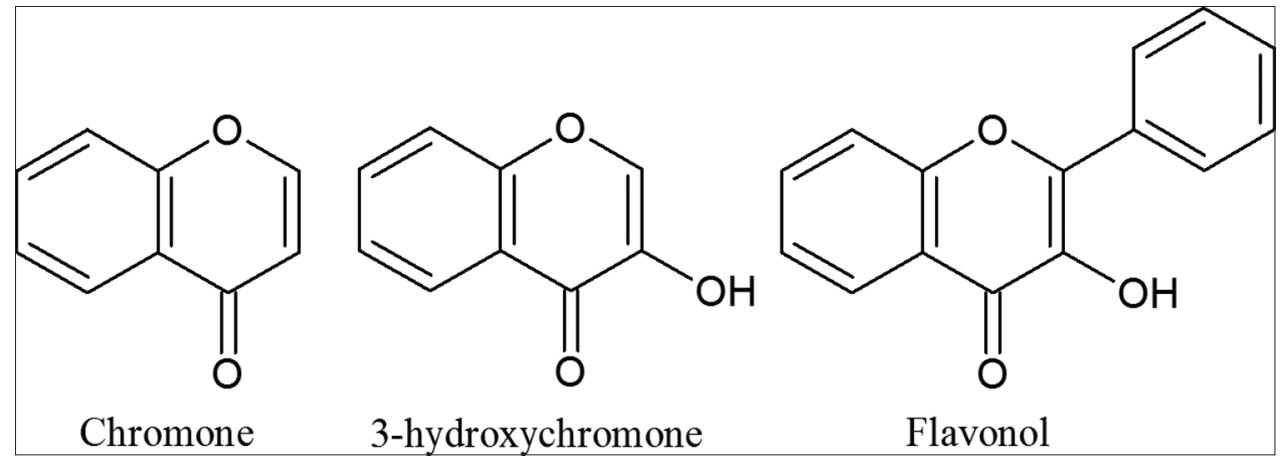

Fig. 1: Chromone core, 3-hydroxy chromone and flavonol<smiles>[R]c1ccc(C(=O)CC)c(O)c1</smiles>

Fig. 2: Synthesis of substituted chalcones and 3-hydroxy chromones (D1-D30)

General procedure for synthesis of 3-hydroxy chromone derivatives (Fig. 2, D1-D30)

Hydrogen peroxide $(4 \mathrm{ml}, 35 \%)$ added to a mixture of chalcone $(10 \mathrm{mmol})$ in methanol $(70 \mathrm{ml})$ and dilute sodium hydroxide $(35 \mathrm{ml}, 5 \%)$ and cooled in ice bath. The solution was stirred for $5 \mathrm{~h}$ at $0-5^{\circ} \mathrm{C}$ and then for $16 \mathrm{~h}$ at room temperature. After completion of the reaction (monitored by TLC), the reaction mixture was poured into ice water and acidified with dilute hydrochloric acid. The precipitate was collected by filtration, washed with water and recrystallized with suitable solvent [14]

3-hydroxy-2-(3-nitrophenyl)-chromone (D1) Yield 85\%; m.p. 158$160^{\circ} \mathrm{C}$; IR (KBr) U : 3647.83 (O-H str), 3080.32 (C-H str), 1716.65 (C=0 str), 1608.63 (C=C str), 1529.55 ( $\mathrm{N}=0 \mathrm{str}), 1309.24$ (C-N str), 1298.09 (C-0 str) $\mathrm{cm}^{-1} ;{ }^{1} \mathrm{H}$ NMR $\left(\mathrm{CDCl}_{3}\right) \delta: 6.971(\mathrm{~s}, 1 \mathrm{H}-\mathrm{OH}), 7.792$ (dd, J=7.7, 1.5, $1 \mathrm{H}-\mathrm{H} 5), 7.063$ (m, 1H-H6), $7.482(\mathrm{~m}, 1 \mathrm{H}-\mathrm{H} 7), 7.047$ (dd, J=8.2, 1.7, $1 \mathrm{H}-$ H8), 7.660 (m, 1H-H2'), 7.265 (m, 1H-H4'), 7.467 (m, 1H-H5'), 7.732 (m, 1H-H6') ppm; mass m/z: 283.2304 (M-1).

2-(4-chlorophenyl)-3-hydroxy-chromone (D2) yield: 92\%; m.p. 176$178^{\circ} \mathrm{C}$; IR (KBr) v : 3630.03 (O-H str), 3049.46 (C-H str), 1718.23 (C=0 str), 1591.27 ( $\mathrm{C}=\mathrm{C}$ str), 1294.24 (C-0 str), 785.03 (C-Cl str) cm ${ }^{-1}$; ${ }^{1} \mathrm{H} \mathrm{NMR}\left(\mathrm{CDCl}_{3}\right)$ 8: 7.345 (s, 1H-OH), 8.146 (dd, J=8.7, 6.3, 1H-H5), 7.568 (m, 1H-H6), 7.490 (m, 1H-H7), 7.656 (dd, J=9.6, 2.6, 1H-H8), 7.943 (m, 2H-H2', H6'), 7.797 (m, 2H-H3', H5') ppm; mass m/z: 272.6810 (M-1).

3-hydroxy-2-phenyl-chromone (D3) yield: 76\%; 196-198 ${ }^{\circ} \mathrm{C}$; IR (KBr) $v_{\max }: 3628.10$ (O-H str), 3070.68 (C-H str), 1716.65 (C=0 str), 1608.63 $\left(\mathrm{C}=\mathrm{C}\right.$ str), $1286.52(\mathrm{C}-\mathrm{O} \mathrm{str}) \mathrm{cm}^{-1} ;{ }^{1} \mathrm{H}$ NMR $\left(\mathrm{CDCl}_{3}\right) \delta: 7.401(\mathrm{~s}, 1 \mathrm{H}-\mathrm{OH})$, 8.256 (dd, J=8.7, 6.3, 1H-H5), 7.260 (m, 1H-H6), 7.476 (m, 1H-H7), 7.093 (dd, J=9.6, 2.6, 1H-H8), 7.555 (m, 2H-H2', H6'), 7.416 (m, 2H-H3', H5'), 7.405 (m, 1H-H4') ppm; mass m/z: 238.2402 (M-1).

3-hydroxy-2-(4-methylphenyl)-chromone (D4) yield: 81\%; 202-204 ${ }^{\circ} \mathrm{C}$; IR (KBr) v max : 3618.46 (O-H str), 3072.60, 2850.79 (C-H str), 1716.65 (C=0 str), 1606.70 (C=C str), 1280.73 (C-O str) cm ${ }^{-1} ;{ }^{1} \mathrm{H}$ NMR (CDCl3) $\delta$ : 6.955 (s, 1H-OH), 7.922 (dd, J=8.7, 6.3, 1H-H5), 6.940 (m, 1H-H6), 7.411 (m, 1H-H7), 6.925 (dd, J=9.6, 2.6, 1H-H8), 7.569 (m, 2H-H2', H6'), 7.168 (m, 2H-H3', H5'), 3.743 (s, 3H-CH ${ }_{3}$ ) ppm; mass m/z: 252.2601 (M-1).

2-(4-bromophenyl)-3-hydroxy-chromone (D5) yield: 93\%; m.p. 284$286^{\circ} \mathrm{C}$; IR (KBr) v : 3632.53 (O-H str), 3066.82 (C-H str), 1749.44
(C=0 str), 1568.13 ( $\mathrm{C}=\mathrm{C}$ str), 1298.09 ( $\mathrm{C}-0$ str), 625.18 (C-Br str) $\mathrm{cm}^{-1}$; ${ }^{1} \mathrm{H} \mathrm{NMR}$ (CDCl3) $\delta: 7.369$ (s, 1H-OH), 8.055 (dd, J=8.7, 6.3, 1H-H5), 7.355 (m, 1H-H6), 7.644 (m, 1H-H7), 7.341 (dd, J=9.6, 2.6, 1H-H8), 7.676 (m, 2H-H2', H6'), 7.667 (m, 2H-H3', H5') ppm; mass m/z: 317.1304 (M-1).

2-(2-chlorophenyl)-3-hydroxy-chromone (D6) yield: 72\%; m.p. 185$187^{\circ} \mathrm{C}$; IR (KBr) $\mathrm{v}_{\max }: 3630.03$ (O-H str), 3061.03 (C-H str), 1716.65 (C=0 str), 1587.42 (C=C str), 1296.84 (C-O str), 784.72 (C-Cl str) cm ${ }^{-1}$; ${ }^{1} \mathrm{H} \mathrm{NMR}\left(\mathrm{CDCl}_{3}\right)$ 8: $7.031(\mathrm{~s}, 1 \mathrm{H}-\mathrm{OH}), 7.952$ (dd, J=7.6, 1.7, 1H-H5), 7.241 (m, 1H-H6), 7.535 (m, 1H-H7), 7.096 (dd, J=8.3, 1.5, 1H-H8), 7.465 (m, 1H-H3'), 7.259 (m, 1H-H4'), 7.048 (m, 1H-H5'), 7.745 (m, 1H-H6') ppm; mass $\mathrm{m} / \mathrm{z}: 272.6810(\mathrm{M}-1)$.

2-(3-chlorophenyl)-3-hydroxy-chromone (D7) yield: 68\%; m.p. 192$194^{\circ} \mathrm{C}$; IR (KBr) v : 3628.10 (O-H str), 3057.17 (C-H str), 1716.65 (C=0 str), 1587.42 ( $\mathrm{C}=\mathrm{C}$ str), 1282.66 (C-0 str), 783.68 (C-Cl str) cm ${ }^{-1}$;

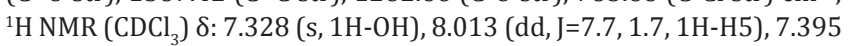
(m, 1H-H6), 7.535 (m, 1H-H7), 7.346 (dd, J=8.3, 1.9, 1H-H8), 7.555 (m, 1H-H2'), 7.377 (m, 1H-H4'), 7.515 (m, 1H-H5'), 7.788 (m, 1H-H6') ppm; mass m/z: $272.6801(\mathrm{M}-1)$

2-(4-fluorophenyl)-3-hydroxy-chromone (D8) yield: 88\%; m.p. 275$277^{\circ} \mathrm{C}$; IR (KBr) v : 3628.10 (O-H str), 3066.82 (C-H str), 1715.72 (C=0 str), 1587.42 ( $\mathrm{C}=\mathrm{C}$ str), 1388.75 (C-F str), 1296.16 (C-0 str) cm ${ }^{-1}$; ${ }^{1} \mathrm{H} \mathrm{NMR}\left(\mathrm{CDCl}_{3}\right) \delta: 7.379(\mathrm{~s}, 1 \mathrm{H}-\mathrm{OH}), 8.003(\mathrm{dd}, \mathrm{J}=8.7,6.3,1 \mathrm{H}-\mathrm{H} 5), 7.281$ (m, 1H-H6), 7.024 (m, 1H-H7), 7.115 (dd, J=9.6, 2.6, 1H-H8), 7.607 (m, 2H-H2', H6'), 7.400 (m, 2H-H3', H5') ppm; mass m/z: 256.2206 (M-1).

2-(3-chlorophenyl)-3,7-dihydroxy-chromone $\quad$ (D9) yield: 75\%;

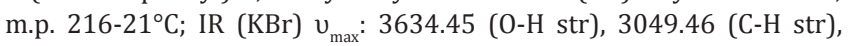
1714.72 (C=0 str), 1573.91 ( $\mathrm{C}=\mathrm{C}$ str), 1294.45 (C-0 str), 783.10 (C-Cl str) $\mathrm{cm}^{-1} ;{ }^{1} \mathrm{H} \mathrm{NMR}\left(\mathrm{CDCl}_{3}\right) \delta: 7.412(\mathrm{~s}, 2 \mathrm{H}-\mathrm{OH}), 8.023(\mathrm{dd}, \mathrm{J}=8.6,6.4,1 \mathrm{H}-$ H5), 7.424 (td, J=8.3, 2.2, 1H-H6), 7.409 (dd, J=9.6, 2.2, 1H-H8), 7.559 (m, 1H-H2'), 7.468 (m, 1H-H4'), 7.493 (m, 1H-H5'), 7.761 (m, 1H-H6') ppm; mass m/z: 288.6801 (M-1).

2-(3-bromophenyl)-3-hydroxy-chromone (D10) yield: 90\%; m.p. 318$320^{\circ} \mathrm{C}$; IR (KBr) v max $_{\operatorname{mo}}: 3647.39$ (O-H str), 3062.96 (C-H str), 1714.72 (C=0 str), 1596.20 (C=C str), 1294.95 (C-O str), 628.30 (C-Br str) cm ${ }^{-1}$; ${ }^{1} \mathrm{H} \mathrm{NMR}\left(\mathrm{CDCl}_{3}\right.$ ) $\delta: 7.439$ (s, 2H-OH), 8.120 (dd, J=8.6, 6.4, 1H-H5), 7.422 (m, 1H-H6), 7.417 (m, 1H-H7), 7.405(dd, J=9.6, 2.2, 1H-H8), 7.562 (m, 
1H-H2'), 7.470 (m, 1H-H4'), 7.490 (m, 1H-H5'), 7.768 (m, 1H-H6') ppm; mass m/z: $317.1302(\mathrm{M}-1)$.

2-(3,4-dimethoxyphenyl)-3-hydroxy-chromone (D11) yield: 80\%; m.p. 287-289 ${ }^{\circ}$; IR (KBr) v : 3639.68 (O-H str), 3078.39, 2841.15 (C-H str), 1716.01 ( $\mathrm{C}=0 \mathrm{str}$ ), 1598.99 ( $\mathrm{C}=\mathrm{C}$ str), 1292.31 (C-0 str), 1028.06 (C-O-C) $\mathrm{cm}^{-1} ;{ }^{1} \mathrm{H} \mathrm{NMR}\left(\mathrm{CDCl}_{3}\right) \delta: 6.937(\mathrm{~s}, 1 \mathrm{H}-\mathrm{OH}), 7.938 \mathrm{dd}, \mathrm{J}=7.4,1.6$, $1 \mathrm{H}-\mathrm{H} 5), 7.167$ (m, 1H-H6), 7.532 (m, 1H-H7), 7.029 (dd, J=8.4, 1.6, 1HH8), 7.501 (m, 1H-H2'), 3.964 (s, 6H-OCH3), 7.013 (m, 1H-H5'), 7.865 (m, 1H-H6') ppm; mass m/z: 298.2910 (M-1)

3,7-dihydroxy-2-(4-methylphenyl)-chromone (D12) yield: 72\%; m.p. 256-258 ${ }^{\circ} \mathrm{C}$; IR (KBr) v : 3618.46 (O-H str), 3026.31, 2852.72 (C-H str), 1716.65 (C=0 str), 1589.34 (C=C str), 1284.59 (C-0 str) cm ${ }^{-1}$; ${ }^{1} \mathrm{H}$ NMR $\left(\mathrm{CDCl}_{3}\right) \delta: 7.180$ (s, 2H-OH), 7.902 (dd, J=8.7, 6.3, 1H-H5), 7.159 (td, J=8.3, 2.2, 1H-H6), 6.800 (dd, J=9.6, 2.6, 1H-H8), 7.799 (m, 2H-H2', H6'), 7.474 (m, 2H-H3', H5'), 3.837 (s, 3H-CH3) ppm; mass m/z: 268.2601 (M-1).

3-hydroxy-2-(3-methylphenyl)-chromone (D13) yield: 78\%; m.p. 224$226^{\circ} \mathrm{C}$; IR (KBr) v $v_{\text {max }}: 3639.68$ (O-H str), 3026.31, 2999.31 (C-H str), 1716.65 (C=0 str), 1600.92 (C=C str), 1271.09 (C-O str) cm ${ }^{-1} ;{ }^{1} \mathrm{H}$ NMR $\left(\mathrm{CDCl}_{3}\right)$ 8: $7.313(\mathrm{~s}, 1 \mathrm{H}-\mathrm{OH}), 8.066(\mathrm{dd}, \mathrm{J}=7.7,1.7,1 \mathrm{H}-\mathrm{H} 5), 7.457(\mathrm{~m}, 1 \mathrm{H}-$ H6), 7.769 (m, 1H-H7), 7.330 (dd, J=8.3, 1.9, 1H-H8), 7.789 (m, 1H-H2'), 7.438 (m, 1H-H4'), 7.493 (m, 1H-H5'), 7.809 (m, 1H-H6'), 3.322 (s, 3HCH3) ppm; mass m/z: 252.2601 (M-1).

3,7-dihydroxy-2-(3-nitrophenyl)-chromone (D14) yield: 77\%; m.p. 244-246 ${ }^{\circ} \mathrm{C}$; IR (KBr) $v_{\text {max }}: 3647.83$ (O-H str), 3080.32 (C-H str), 1710.86 ( $\mathrm{C}=0 \mathrm{str}$ ), 1598.99 ( $\mathrm{C}=\mathrm{C}$ str), 1529.55 ( $\mathrm{N}=0 \mathrm{str}$ ), 1309.24 (C-N str) 1282.99 (C-0 str) cm ${ }^{-1} ;{ }^{1} \mathrm{H}$ NMR $\left(\mathrm{CDCl}_{3}\right) \delta: 7.415(\mathrm{~s}, 2 \mathrm{H}-\mathrm{OH}), 8.016$ (dd, J=8.6, 6.4, 1H-H5), 7.427 (td, J=8.3, 2.2, 1H-H6), 7.405 (dd, J=9.6, 2.2, 1H-H8), 7.558 (m, 1H-H2'), 7.463 (m, 1H-H4'), 7.491 (m, 1H-H5'), 7.767 (m, 1H-H6') ppm; mass m/z: 299.2304 (M-1).

2-(4-chlorophenyl)-3,7-dihydroxy-chromone (D15) yield: 75\%; m.p. 188-190 ${ }^{\circ}$; IR (KBr) $v_{\max }: 3630.03$ (O-H str), 3093.82 (C-H str), 1718.23 (C=0 str), 1593.20 (C=C str), 1282.66 (C-0 str), 761.88 (C-Cl str) $\mathrm{cm}^{-1} ;{ }^{1} \mathrm{H}$ NMR $\left(\mathrm{CDCl}_{3}\right) \delta: 7.183(\mathrm{~s}, 2 \mathrm{H}-\mathrm{OH}), 7.907(\mathrm{dd}, \mathrm{J}=8.7,6.3,1 \mathrm{H}-$ H5), 7.155 (td, J=8.3, 2.2, 1H-H6), 6.802 (dd, J=9.6, 2.6, 1H-H8), 7.798 (m, 2H-H2', H6'), 7.473 (m, 2H-H3', H5') ppm; mass m/z: 288.6810 (M-1).

3,7-dihydroxy-2-phenyl-chromone (D16) yield: 72\%; m.p. 262-264 ${ }^{\circ} \mathrm{C}$; IR (KBr) v $v_{\max }: 3628.10$ (O-H str), 3080.32 (C-H str), 1716.65 (C=0 str), 1604.77 ( C=C str), 1294.24 (C-0 str) cm ${ }^{-1} ;{ }^{1} \mathrm{H}$ NMR $\left(\mathrm{CDCl}_{3}\right) \delta: 7.405$ (s, 2H-OH), 8.257 (dd, J=8.7, 6.3, 1H-H5), 7.263 (td, J=8.4, 2.6, 1H-H6), 7.099 (dd, J=9.6, 2.6, 1H-H8), 7.551 (m, 2H-H2', H6'), 7.419 (m, 2H-H3', H5'), 7.405 (m, 1H-H4') ppm; mass m/z: 254.2306 (M-1).

2-(4-bromophenyl)-3,7-dihydroxy-chromone (D17) yield: 92\%; m.p. 326-328 ${ }^{\circ}$; IR (KBr) $v_{\max }: 3639.68$ (O-H str), 3064.89 (C-H str), 1714.72 (C=0 str), 1596.20 ( $\mathrm{C}=\mathrm{C}$ str), 1293.17 (C-O str), 644.22 (C-Br str) $\mathrm{cm}^{-1} ;{ }^{1} \mathrm{H} \mathrm{NMR}\left(\mathrm{CDCl}_{3}\right) \delta: 7.224(\mathrm{~s}, 2 \mathrm{H}-\mathrm{OH}), 8.008(\mathrm{dd}, \mathrm{J}=8.7,6.3,1 \mathrm{H}-$ H5), 6.543 (td, J=8.3, 2.7, 1H-H6), 6.439 (dd, J=9.6, 2.6, 1H-H8), 7.554 (m, 2H-H2', H6'), 7.354 (m, 2H-H3', H5') ppm; mass m/z: 333.1301 (M-1).

2-(4-fluorophenyl)-3,7-dihydroxy-chromone (D18) yield: 88\%; m.p. 272-274 ${ }^{\circ}$; IR (KBr) v max $_{\text {max }} 3628.10$ (O-H str), 3066.82 (C-H str), 1716.01 (C=0 str), 1578.42 (C=C str), 1398.39 (C-F str), 1271.09 (C-O str) $\mathrm{cm}^{-1} ;{ }^{1} \mathrm{H} \mathrm{NMR}\left(\mathrm{CDCl}_{3}\right) \delta: 6.971(\mathrm{~s}, 2 \mathrm{H}-\mathrm{OH}), 8.004(\mathrm{dd}, \mathrm{J}=8.7,6.3,1 \mathrm{H}-$ H5), 7.262 (td, J=8.3, 2.7, 1H-H6), 6.795 (dd, J=9.6, 2.6, 1H-H8), 7.739 (m, 2H-H2', H6'), 7.357 (m, 2H-H3', H5') ppm; mass m/z: 272.2201 (M-1).

3-hydroxy-2-(2-nitrophenyl)-chromone (D19) yield: 70\%; m.p. 285$287^{\circ} \mathrm{C}$; IR (KBr) v $_{\text {max }}: 3608.81$ (O-H str), 3032.10 (C-H str), 1710.22 (C=O str), 1598.99 (C=C str), 1529.55 ( $\mathrm{N}=0 \mathrm{str}$ ), 1340.53 (C-N str), 1288.45
(C-0 str) $\mathrm{cm}^{-1} ;{ }^{1} \mathrm{H} \mathrm{NMR}\left(\mathrm{CDCl}_{3}\right) \delta: 6.975(\mathrm{~s}, 1 \mathrm{H}-\mathrm{OH}), 7.962$ (dd, J=7.6, 1.7, 1H-H5), 7.174 (m, 1H-H6), 7.536 (m, 1H-H7), 7.101 (dd, J=8.3, 1.5, 1HH8), 7.502 (m, 1H-H3'), 7.260 (m, 1H-H4'), 7.062 (m, 1H-H5'), 7.743 (m, 1H-H6') ppm; mass m/z: 283.2312 (M-1)

2-(3,4-dimethoxyphenyl)-3,7-dihydroxy-chromone (D20) yield: 72\%; m.p. 258-260 ${ }^{\circ}$; IR (KBr) $v_{\max }$ : $3628.10(\mathrm{O}-\mathrm{H}$ str), 3078.39, 2995.45 (C-H str), 1716.65 ( $\mathrm{C}=0 \mathrm{str}$ ), 1575.84 ( $\mathrm{C}=\mathrm{C}$ str), 1286.52 (C-O str), 1026.13 (C-O-C) cm ${ }^{-1} ;{ }^{1} \mathrm{H} \mathrm{NMR}\left(\mathrm{CDCl}_{3}\right) \delta: 6.998$ (s, $\left.2 \mathrm{H}-\mathrm{OH}\right), 7.792$ (dd, $\mathrm{J}=8.8,6.2,1 \mathrm{H}-\mathrm{H} 5), 6.842$ (td, J=8.1, 2.4, 1H-H6), 6.804 (dd, J=9.5, 2.5, $1 \mathrm{H}-\mathrm{H} 8), 7.480$ (m, 1H-H2'), $3.883\left(\mathrm{~s}, 6 \mathrm{H}-\mathrm{OCH}_{3}\right), 7.108$ (m, 1H-H5'), 7.747 (m, 1H-H6') ppm; mass m/z: 314.2814 (M-1).

3,7-dihydroxy-2-(4-nitrophenyl)-chromone (D21) yield: 75\%; m.p. 236-238 ${ }^{\circ}$ C; IR (KBr) v $v_{\text {max }}$ : 3618.32 (O-H str), 3032.62 (C-H str), 1716.65 (C=0 str), 1587.42 (C=C str), 1529.42 ( $\mathrm{N}=0 \mathrm{str}$ ), 1311.59 (C-N str), 1288.45 (C-O str) cm ${ }^{-1} ;{ }^{1} \mathrm{H} \mathrm{NMR}\left(\mathrm{CDCl}_{3}\right) \delta: 6.983(\mathrm{~s}, 2 \mathrm{H}-\mathrm{OH}), 7.822$ (dd, J=8.7, 6.3, 1H-H5), 6.968 (td, J=8.3, 2.7, 1H-H6), 6.587 (dd, J=9.6, 2.6, 1H-H8), 7.561 (m, 2H-H2', H6'), 7.282 (m, 2H-H3', H5') ppm; mass m/z: 299.2301 (M-1).

3,7-dihydroxy-2-(2-nitrophenyl)-chromone (D22) yield: 71\%; m.p. 240-242-C; IR (KBr) $v_{\text {max }}$ : 3614.60 (O-H str), 3032.10 (C-H str), 1716.65 (C=0 str), 1598.99 (C=C str), 1529.55 ( $\mathrm{N}=0 \mathrm{str}$ ), 1340.53 (C-N str), 1244.09 (C-O str) cm ${ }^{-1} ;{ }^{1} \mathrm{H} \mathrm{NMR}\left(\mathrm{CDCl}_{3}\right) \delta: 6.973(\mathrm{~s}, 2 \mathrm{H}-\mathrm{OH}), 7.965$ (dd, J=7.3, 1.9, 1H-H5), 7.177 (td, J=8.1, 2.6, 1H-H6), 7.107 (dd, J=8.4, 1.8, 1H-H8), 7.503 (m, 1H-H3'), 7.264 (m, 1H-H4'), 7.069 (m, 1H-H5'), 7.742 (m, 1H-H6') ppm; mass m/z: 299.2304 (M-1).

3-hydroxy-2-(3-hydroxy-4-methoxyphenyl)-chromone (D23) yield: 80\%; m.p. 256-258 ${ }^{\circ}$; IR (KBr) $v_{\text {max }} 3628.10$ (O-H str), 3072.39, 2991.59 (C-H str), 1716.65 (C=O str), 1541.12 (C=C str), 1276.88 (C-0 str), 1022.27 (C-O-C) cm ${ }^{-1} ;{ }^{1} \mathrm{H}$ NMR ( $\left.\mathrm{CDCl}_{3}\right) \delta: 6.987$ (s, 2H-OH), 7.919 (dd, J=7.1, 1.9, 1H-H5), $7.086(\mathrm{~m}, 1 \mathrm{H}-\mathrm{H} 6), 7.582(\mathrm{~m}, 1 \mathrm{H}-\mathrm{H} 7), 7.083$ (dd, $\mathrm{J}=8.4,1.5,1 \mathrm{H}-\mathrm{H} 8), 7.516\left(\mathrm{~m}, 1 \mathrm{H}-\mathrm{H} 2\right.$ '), $3.982\left(\mathrm{~s}, 3 \mathrm{H}-\mathrm{OCH}_{3}\right), 7.032(\mathrm{~m}, 1 \mathrm{H}-$ H5'), 7.855 (m, 1H-H6') ppm; mass m/z: 284.2602 (M-1).

2-(3-bromophenyl)-3,7-dihydroxy-chromone (D24) yield: 94\%; m.p. $324-326^{\circ} \mathrm{C}$; IR (KBr) $\mathrm{v}_{\max }: 3608.81$ (O-H str), 3064.89 (C-H str), 1716.65 (C=0 str), 1570.06 ( $\mathrm{C}=\mathrm{C}$ str), 1261.45 (C-0 str), 661.27 (C-Br str) $\mathrm{cm}^{-1} ;{ }^{1} \mathrm{H}$ NMR $\left(\mathrm{CDCl}_{3}\right) \delta: 7.189(\mathrm{~s}, 2 \mathrm{H}-\mathrm{OH}), 7.813(\mathrm{dd}, \mathrm{J}=8.2,6.3,1 \mathrm{H}-$ H5), 6.632 (td, J=8.5, 2.4, 1H-H6), 6.479 (dd, J=9.7, 2.8, 1H-H8), 7.551 (m, 1H-H2'), 7.203 (m, 1H-H4'), 7.454 (m, 1H-H5'), 7.742 (m, 1H-H6') ppm; mass m/z: 333.1304 (M-1).

2-(2-chlorophenyl)-3,7-dihydroxy-chromone (D25) yield: 66\%; m.p. 272-274 ${ }^{\circ} \mathrm{C}$; IR (KBr) v max $: 3628.10$ (O-H str), 3064.89 (C-H str), 1716.65 (C=0 str), 1541.12 (C=C str), 1281.09 (C-0 str), 756.10 (C-Cl str) $\mathrm{cm}^{-1},{ }^{1} \mathrm{H}$ NMR $\left(\mathrm{CDCl}_{3}\right) \delta: 6.991(\mathrm{~s}, 2 \mathrm{H}-\mathrm{OH}), 8.081$ (dd J=7.3, 1.9, $1 \mathrm{H}-$ H5), 7.177 (td J=8.1, 2.6, 1H-H6), 7.132 (dd, J=8.4, 1.8, 1H-H8), 7.535 (m, 1H-H3'), 7.284 (m, 1H-H4'), 7.023 (m, 1H-H5'), 7.754 (m, 1H-H6') ppm; mass m/z: 288.6800 (M-1).

3,7-dihydroxy-2-(4-methoxyphenyl)-chromone (D26) yield: 86\%; m.p. 296-298 ${ }^{\circ}$; IR (KBr) $v_{\max }: 3628.10$ (O-H str), 3072.39, 2985.81 (C-H str), 1701.22 ( $\mathrm{C}=0$ str), 1541.12 ( $\mathrm{C}=\mathrm{C}$ str), 1294.24 (C-0 str), 1033.85 $(\mathrm{C}-\mathrm{O}-\mathrm{C}) \mathrm{cm}^{-1} ;{ }^{1} \mathrm{H}$ NMR $\left(\mathrm{CDCl}_{3}\right) \delta: 6.957(\mathrm{~s}, 2 \mathrm{H}-\mathrm{OH}), 7.878(\mathrm{dd}, \mathrm{J}=8.5$, 6.1, 1H-H5), 7.262 (td, J=8.7, 2.6, 1H-H6), 6.890 (dd, J=9.6, 2.8, 1H-H8), 7.820 (m, 2H-H2', H6'), 7.467 (m, 2H-H3', H5'), 3.835 (s, 3H- $\mathrm{OCH}_{3}$ ) ppm; mass m/z: $284.2610(\mathrm{M}-1)$.

2-(furan-2-yl)-3,7-dihydroxy-chromone (D27) Yield: 92\%; m.p. 316$318^{\circ} \mathrm{C}$; IR (KBr) v max $: 3612.67$ (O-H str), 3076.46 (C-H str), 1716.65 ( $\mathrm{C}=0$ str), 1541.05 ( $\mathrm{C}=\mathrm{C}$ str), 1265.30 (C-O str), 1020.34 (C-O-C) $\mathrm{cm}^{-1} ;{ }^{1} \mathrm{H}$ NMR $\left(\mathrm{CDCl}_{3}\right) \delta: 7.264$ (s, $\left.2 \mathrm{H}-\mathrm{OH}\right), 7.815$ (dd, J=8.8, 6.6, $1 \mathrm{H}-$ H5), 6.731 (td, J=8.4, 2.9, 1H-H6), 6.724 (dd, J=9.5, 2.6, 1H-H8), 7.538 (m, 1H-H3'), 7.439 (m, 1H-H4'), 7.469 (m, 1H-H5') ppm; mass m/z: 244.1906 (M-1). 
3-hydroxy-2-(4-methoxyphenyl)-chromone (D28) yield: 85\%; m.p. 265-267º ; IR (KBr) v max $_{\text {ax }} 3628.10$ (O-H str), 3066.82, 2947.23 (C-H str), 1716.65 ( $\mathrm{C}=0$ str), 1541.12 ( $\mathrm{C}=\mathrm{C}$ str), 1296.16 (C-0 str), 1022.27 (C-O-C) $\mathrm{cm}^{-1} ;{ }^{1} \mathrm{H}$ NMR $\left(\mathrm{CDCl}_{3}\right) \delta: 6.993(\mathrm{~s}, 1 \mathrm{H}-\mathrm{OH}), 7.931$ (dd, J=7.6, 1.8, $1 \mathrm{H}-\mathrm{H} 5), 7.127$ (m, 1H-H6), 7.625 (m, 1H-H7), 7.110 (dd, J=8.3, 1.7, 1HH8), 7.638 (m, 2H-H2', H6'), 7.249 (m, 2H-H3', H5'), 3.863 (s, 3H- $\mathrm{OCH}_{3}$ ) ppm; mass m/z: 268.2609 (M-1).

2-(furan-2-yl)-3-hydroxy-chromone (D29) yield: 93\%; m.p. 310-312 ${ }^{\circ} \mathrm{C}$; IR (KBr) v $v_{\max }: 3649.32$ (O-H str), 3095.75 (C-H str), 1716.65 (C=O str), 1568.13 (C=C str), 1298.09 (C-O str), 1016.49 (C-O-C) cm ${ }^{-1} ;{ }^{1} \mathrm{H}$ NMR $\left(\mathrm{CDCl}_{3}\right)$ \&: $6.53(\mathrm{~s}, 1 \mathrm{H}-\mathrm{OH}), 7.930(\mathrm{dd}, \mathrm{J}=7.8,1.6,1 \mathrm{H}-\mathrm{H} 5), 7.260(\mathrm{~m}, 1 \mathrm{H}-$ H6), 7.572 (m, 1H-H7), 7.027 (dd, J=8.3, 1.9, 1H-H8), 7.542 (m, 1H-H3'), 7.125 (m, 1H-H4'), 7.491 (m, 1H-H5')ppm; mass m/z: 228.2010 (M-1).

3-hydroxy-2-(4-nitrophenyl)-chromone (D30) yield: 84\%; m.p. 254$256^{\circ} \mathrm{C}$; IR (KBr) v max $_{\operatorname{ma}}$ : 3614.60 (O-H str), 3032.10 (C-H str), 1714.72 ( $\mathrm{C}=0$ str), 1566.20 ( $\mathrm{C}=\mathrm{C}$ str), 1539.20 ( $\mathrm{N}=0 \mathrm{str}), 1336.67$ (C-N str), 1269.16 (C-O str) cm ${ }^{-1} ;{ }^{1} \mathrm{H}$ NMR $\left(\mathrm{CDCl}_{3}\right) \delta: 6.996(\mathrm{~s}, 1 \mathrm{H}-\mathrm{OH}), 7.963$ (dd, J=8.7, 6.3, 1H-H5), 7.281 (m, 1H-H6), 7.028 (m, 1H-H7), 7.046 (dd, J=9.6, 2.6, 1H-H8), 7.608 (m, 2H-H2', H6'), 7.469 (m, 2H-H3', H5') ppm; mass m/z: $283.2310(\mathrm{M}-1)$

\section{In vitro antioxidant study}

The synthesized compounds were evaluated for their in vitro antioxidant activity by DPPH radical scavenging method. DPPH is a stable free radical that can accept an electron or hydrogen radical to become a stable diamagnetic molecule. Due to its odd electron, the methanolic solution of DPPH shows a strong absorption band at $517 \mathrm{~nm}$. The DPPH radical reacts with various electron donating molecules (reducing agents or antioxidants). When electrons become paired off, bleaching of the DPPH solution occurs. This results in the formation of the colorless 1,1-diphenyl-1-picryl hydrazine. Reduction of the DPPH radicals can be estimated quantitatively by measuring the decrease in absorbance at $517 \mathrm{~nm}$ [15]. A radical scavenging antioxidant reacts with DPPH stable free radical and converts to DPPH-H. The change in the absorbance produced in this reaction has been used to measure antioxidant properties.

A stock solution of DPPH $(1.3 \mathrm{mg} / \mathrm{ml})$ in methanol was prepared. A stock solution of DPPH $100 \mu \mathrm{l}$ was added to $3.0 \mathrm{ml}$ of methanol and absorbance was recorded at $517 \mathrm{~nm}$. The various concentrations of compounds $(20,40,60,80$, and $100 \mu \mathrm{g} / \mathrm{ml})$ were prepared. All sample solutions $1.0 \mathrm{ml}$ each is diluted with $3.0 \mathrm{ml}$ with methanol, and $100 \mu \mathrm{l}$ of stock solution of DPPH was added. Test tubes were kept for $30 \mathrm{~min}$ in light to complete the reaction. After $30 \mathrm{~min}$, the absorbance of each test tube was recorded at $517 \mathrm{~nm}$ on ultravioletvisible (UV-VIS) spectrophotometer against methanol as a blank [16]. Control experiment was carried out with solvent only, and ascorbic acid was used as reference standard. All the measurements were performed in triplicate, and the mean of triplicate measurements was used to calculate the percentage reduction of DPPH by the following formula:

$\%$ scavenging $=\frac{\text { Absorbance of control }}{\text { Absorbance of test sample }} \times 100$

Where,

Control is absorbance of a DPPH solution without compound;

Test is the absorbance of the test compound with DPPH.

The degree of discoloration indicates the free radical scavenging efficiency of the compound. The effective concentration of sample required to scavenge DPPH radical by $50 \%\left(\mathrm{IC}_{50}\right.$ value) was obtained by linear regression analysis of dose-response curve plotted between $\%$ inhibition and concentrations.
In vitro antibacterial activity

The antibacterial activity of synthesized compounds was measured using the agar cup method. Nutrient agar (Himedia) was prepared and sterilized at $100 \mathrm{kPa}$ for $15 \mathrm{~min}$ in the autoclave. It was allowed to cool below $45^{\circ} \mathrm{C}$ and seeded with turbid suspension of test bacteria separately, prepared from $24 \mathrm{~h}$ old slant cultures. $3 \%$ inocula were used every time. The bacterial cultures selected were, two Gram-negative cultures, namely, Escherichia coli, S. typhi and two Gram-positive cultures, namely, Staphylococcus aureus, Bacillus subtilis. This seeded preparation was then poured into sterile petri plate under the aseptic condition and allowed to solidify.

Cups of $10 \mathrm{~mm}$ diameter were borered in the agar plate with sterile cork borer, $100 \mu \mathrm{l}$ of compound solution prepared in $1 \%$ dimethyl sulfoxide (DMSO) was added in the cup under an aseptic condition with the help of micropipette. A volume of $100 \mu$ l of DMSO was also placed in one of the cups as blank (negative control). A standard antibiotic disk impregnated with 10 units of Penicillin was also placed on the seeded nutrient agar surface as a standard reference antibiotic (positive control).

The plates were kept in the refrigerator for 15 min to allow diffusion of the compound from the agar cup into the medium. Then, the plates were shifted to the incubator at $37^{\circ} \mathrm{C}$ and incubated for $24 \mathrm{~h}$ [17].

After incubation plates were observed for the zone of inhibition of bacterial growth around the agar cup. Results were recorded by measuring the zone of inhibition in millimeter $(\mathrm{mm})$ using zone reader.

\section{In vitro antifungal activity}

Antifungal activity of title compounds was performed using the poison plate method. The medium used was Potato dextrose agar (Himedia). The medium was prepared and sterilized at $65 \mathrm{kPa}$ in an autoclave for $15 \mathrm{~min}$. Then the compound to be tested is added to the sterile medium in aseptic condition to get a final concentration of $1 \%$. A plate with DMSO was prepared as blank (negative control) similarly a plate with $1 \%$ griseofulvin was prepared as standard reference plate (positive control).

Aspergillus niger, Penicillium chrysogenum, Fusarium moneliforme, and Aspergillus flavus were selected as test fungal cultures. They were allowed to grow on slant for $48 \mathrm{~h}$ to get profuse sporulation. A volume of $5 \mathrm{ml}$ of 1:100 aqueous solution of Tween 80 was added to the slant and spores were scraped with the help of a nicrome wire loop to form a suspension.

The fungal suspension was spot inoculated on the plates prepared using compound with the help of a nichrome wire loop. The plates were incubated at room temperature for $48 \mathrm{~h}$ [17].

After incubation plates were observed for the growth of inoculated fungi. Results were recorded as the growth of fungi (no antifungal activity), reduced growth of fungi (moderate antifungal activity), and no growth of inoculated fungi (antifungal activity).

\section{RESULTS AND DISCUSSION}

In our laboratory, a facile method has been adopted for the synthesis of 3-hydroxy chromones by base catalyzed cyclization of chalcones in the presence of hydrogen peroxide. The respective chalcones have been synthesized by Claisen-Schmidt condensation of substituted 2-hydroxy acetophenones with substituted aromatic aldehydes using PEG-400 as a recyclable solvent. The reaction was simple and efficient and yields the title compounds almost in pure form. However, the resultant compounds were purified by recrystallization with a suitable solvent. The compounds obtained in good yields ranging from $66 \%$ to $94 \%$. The physicochemical properties of the synthesized compounds are given in Table 1. The structures of the synthesized compounds were confirmed by IR, ${ }^{1} \mathrm{H}$ NMR, and mass spectra. 
Table 1: Physicochemical properties of synthesized derivatives

\begin{tabular}{|c|c|c|c|c|c|c|c|}
\hline Compound code & $\mathbf{R}^{1}$ & Ar & $\begin{array}{l}\text { Molecular } \\
\text { formula }\end{array}$ & $\begin{array}{l}\text { Molecular } \\
\text { weight (g) }\end{array}$ & Yield (\%) & Melting point $\left({ }^{\circ} \mathrm{C}\right)$ & $\mathbf{R}^{\mathbf{f}}$ value* \\
\hline D1 & $\mathrm{H}$ & & $\mathrm{C}_{15} \mathrm{H}_{9} \mathrm{NO}_{5}$ & 283.23 & 85 & $158-160$ & 0.65 \\
\hline D2 & $\mathrm{H}$ & & $\mathrm{C}_{15} \mathrm{H}_{9} \mathrm{ClO}_{3}$ & 272.68 & 92 & $176-178$ & 0.51 \\
\hline D3 & $\mathrm{H}$ & & $\mathrm{C}_{15} \mathrm{H}_{10} \mathrm{O}_{3}$ & 238.24 & 76 & 196-198 & 0.71 \\
\hline D4 & $\mathrm{H}$ & & $\mathrm{C}_{16} \mathrm{H}_{12} \mathrm{O}_{3}$ & 252.26 & 81 & $202-204$ & 0.75 \\
\hline D5 & $\mathrm{H}$ & & $\mathrm{C}_{15} \mathrm{H}_{9} \mathrm{BrO}_{3}$ & 317.13 & 93 & $284-286$ & 0.78 \\
\hline D6 & $\mathrm{H}$ & & $\mathrm{C}_{15} \mathrm{H}_{9} \mathrm{ClO}_{3}$ & 272.68 & 72 & $185-187$ & 0.53 \\
\hline D7 & $\mathrm{H}$ & & $\mathrm{C}_{15} \mathrm{H}_{9} \mathrm{ClO}_{3}$ & 272.68 & 68 & $192-194$ & 0.57 \\
\hline D8 & $\mathrm{H}$ & & $\mathrm{C}_{15} \mathrm{H}_{9} \mathrm{FO}_{3}$ & 256.22 & 88 & $275-277$ & 0.65 \\
\hline D9 & $\mathrm{OH}$ & & $\mathrm{C}_{15} \mathrm{H}_{9} \mathrm{ClO}_{4}$ & 288.68 & 75 & $216-218$ & 0.72 \\
\hline D10 & $\mathrm{H}$ & & $\mathrm{C}_{15} \mathrm{H}_{9} \mathrm{BrO}_{3}$ & 317.13 & 90 & $318-320$ & 0.86 \\
\hline D11 & $\mathrm{H}$ & & $\mathrm{C}_{17} \mathrm{H}_{14} \mathrm{O}_{5}$ & 298.29 & 80 & $287-289$ & 0.82 \\
\hline D12 & $\mathrm{OH}$ & & $\mathrm{C}_{16} \mathrm{H}_{12} \mathrm{O}_{4}$ & 268.26 & 72 & $256-258$ & 0.77 \\
\hline
\end{tabular}


Table 1: Continued

\begin{tabular}{|c|c|c|c|c|c|c|c|}
\hline Compound code & $\mathbf{R}^{1}$ & Ar & $\begin{array}{l}\text { Molecular } \\
\text { formula }\end{array}$ & $\begin{array}{l}\text { Molecular } \\
\text { weight (g) }\end{array}$ & Yield (\%) & Melting point $\left({ }^{\circ} \mathrm{C}\right)$ & $\mathbf{R}^{\mathbf{f}}$ value* \\
\hline D13 & $\mathrm{H}$ & & $\mathrm{C}_{16} \mathrm{H}_{12} \mathrm{O}_{3}$ & 252.26 & 78 & $224-226$ & 0.76 \\
\hline D14 & $\mathrm{OH}$ & & $\mathrm{C}_{15} \mathrm{H}_{9} \mathrm{NO}_{6}$ & 299.23 & 77 & $244-246$ & 0.69 \\
\hline D15 & $\mathrm{OH}$ & & $\mathrm{C}_{15} \mathrm{H}_{9} \mathrm{ClO}_{4}$ & 288.68 & 75 & $188-190$ & 0.76 \\
\hline D16 & $\mathrm{OH}$ & & $\mathrm{C}_{15} \mathrm{H}_{10} \mathrm{O}_{4}$ & 254.23 & 72 & $262-264$ & 0.82 \\
\hline D17 & $\mathrm{OH}$ & & $\mathrm{C}_{15} \mathrm{H}_{9} \mathrm{BrO}_{4}$ & 333.13 & 92 & $326-328$ & 0.71 \\
\hline D18 & $\mathrm{OH}$ & & $\mathrm{C}_{15} \mathrm{H}_{9} \mathrm{FO}_{4}$ & 272.22 & 88 & $272-274$ & 0.79 \\
\hline D19 & $\mathrm{H}$ & & $\mathrm{C}_{15} \mathrm{H}_{9} \mathrm{NO}_{5}$ & 283.23 & 70 & $285-287$ & 0.81 \\
\hline D20 & $\mathrm{OH}$ & & $\mathrm{C}_{17} \mathrm{H}_{14} \mathrm{O}_{6}$ & 314.28 & 72 & $258-260$ & 0.67 \\
\hline D21 & $\mathrm{OH}$ & & $\mathrm{C}_{15} \mathrm{H}_{9} \mathrm{NO}_{6}$ & 299.23 & 75 & $236-238$ & 0.70 \\
\hline D22 & $\mathrm{OH}$ & & $\mathrm{C}_{15} \mathrm{H}_{9} \mathrm{NO}_{6}$ & 299.23 & 77 & $240-242$ & 0.71 \\
\hline D23 & $\mathrm{H}$ & & $\mathrm{C}_{16} \mathrm{H}_{12} \mathrm{O}_{5}$ & 284.26 & 80 & $256-258$ & 0.73 \\
\hline
\end{tabular}

Contd... 
Table 1: Continued

\begin{tabular}{|c|c|c|c|c|c|c|c|}
\hline Compound code & $\mathbf{R}^{1}$ & $\mathrm{Ar}$ & $\begin{array}{l}\text { Molecular } \\
\text { formula }\end{array}$ & $\begin{array}{l}\text { Molecular } \\
\text { weight (g) }\end{array}$ & Yield (\%) & Melting point $\left({ }^{\circ} \mathrm{C}\right)$ & $\mathbf{R}^{\mathrm{f}}$ value* \\
\hline D24 & $\mathrm{OH}$ & $3 r$ & $\mathrm{C}_{15} \mathrm{H}_{9} \mathrm{BrO}_{4}$ & 333.13 & 94 & $324-326$ & 0.83 \\
\hline D25 & $\mathrm{OH}$ & & $\mathrm{C}_{15} \mathrm{H}_{9} \mathrm{ClO}_{4}$ & 288.68 & 66 & $272-274$ & 0.77 \\
\hline D26 & $\mathrm{OH}$ & & $\mathrm{C}_{12} \mathrm{H}_{16} \mathrm{O}_{5}$ & 284.26 & 86 & $296-298$ & 0.75 \\
\hline D27 & $\mathrm{OH}$ & & $\mathrm{C}_{13} \mathrm{H}_{8} \mathrm{O}_{5}$ & 244.19 & 92 & $316-318$ & 0.84 \\
\hline D28 & $\mathrm{H}$ & & $\mathrm{C}_{16} \mathrm{H}_{12} \mathrm{O}_{4}$ & 268.26 & 85 & $265-267$ & 0.74 \\
\hline D29 & $\mathrm{H}$ & & $\mathrm{C}_{13} \mathrm{H}_{8} \mathrm{O}_{4}$ & 228.20 & 93 & $310-312$ & 0.81 \\
\hline D30 & $\mathrm{H}$ & & $\mathrm{C}_{15} \mathrm{H}_{9} \mathrm{NO}_{5}$ & 283.23 & 84 & $254-256$ & 0.73 \\
\hline
\end{tabular}

*Solvent system-4:1 ratio of n-Hexane and ethyl acetate

The IR spectra of final compounds showed an absorption band at 3649.32-3608.81/cm indicative of $\mathrm{O}-\mathrm{H}$ stretching of a phenolic group. The absorption bands in the region of 3095.75-2841.15/cm indicative of C-H stretching and in the region of 1298.09-1244.09/cm corresponds to $\mathrm{C}-\mathrm{O}$ stretching. The absorption band corresponding to a carbonyl group appeared in the region of $1749.44-1701.22 / \mathrm{cm}$. The absorption peaks at $1608.63-1541.05 / \mathrm{cm}$ indicate the $\mathrm{C}=\mathrm{C}$ stretching vibrations. The absorption band representing $\mathrm{N}=\mathrm{O}$ stretching was appeared in the region of 1539.20-1529.42/cm,and those representing C-N stretching was appeared in the region of $1340.53-1309.24 / \mathrm{cm}$. Compounds containing the halogen group, namely, fluoro, chloro and bromo showed an absorption band in the region of $1398.39-1388.75 / \mathrm{cm}, 785.03-$ $756.10 / \mathrm{cm}$ and $661.27-625.18 / \mathrm{cm}$, respectively. The compounds with methoxy substitution exhibited an absorption band in the region of 1033.85-1016.49/cm due to C-O-C stretching vibration.

The NMR spectra of the title compounds showed singlets in the region of $\delta$ 6.937-7.439 due to the protons of the phenolic hydroxyl group. The spectra of the compounds showed singlets in the region of $\delta$ 3.322-3.743 indicative of methyl protons. The compounds containing the methoxy group exhibited characteristic signals in the region of $\delta$ 3.835-3.982. The spectra also exhibited double doublets, triple doublets, and multiplets in the region of $\delta 6.439-8.257$ assignable to aromatic protons in all the NMR spectra of final compounds confirm the structures of title compounds.
The mass spectra of final compounds showed their characteristic molecular ion peak. Thus, the structures of the compounds were confirmed by IR, ${ }^{1} \mathrm{H}$ NMR and mass spectral data.

\section{In vitro antioxidant studies}

The synthesized compounds, 3-hydroxy chromones were evaluated for their in vitro antioxidant properties at 20,40,60, 80, and $100 \mu \mathrm{M}$ by DPPH radical scavenging model. The activity data are presented in Table 2.

The study revealed that all the synthesized 3-hydroxy chromones exhibited potent antioxidant activity with $\mathrm{IC}_{50}$ below $70 \mu \mathrm{M}$. All the synthesized compounds contain 2,3 double bond, one phenolic hydroxyl group and adjacent to that is the presence of $\alpha, \beta$-unsaturated keto group. These structural features were responsible for the presence of antioxidant activity. According to the literature, the 2,3 double bond in conjugation with a 4-oxo function is responsible for electron delocalization from the aromatic ring at the $2^{\text {nd }}$ position. The antioxidant potency is related to structure in terms of electron delocalization of the aromatic nucleus. When these compounds react with free radicals, the phenoxyl radicals produced are stabilized by the resonance effect of the aromatic nucleus. In addition, the $3-\mathrm{OH}$ and 4-oxo functional groups are required for maximum radical scavenging potential [18]. Among the synthesized compounds, the compound 3-hydroxy-2-(3-hydroxy4-methoxyphenyl)-chromone (D23) showed the highest activity with 
Table 2: In vitro antioxidant activity of 3-hydroxy chromones

\begin{tabular}{|c|c|c|c|c|c|c|}
\hline \multirow[t]{3}{*}{$\downarrow$ Compound $\rightarrow$} & \multicolumn{5}{|c|}{ Concentration } & \multirow[t]{3}{*}{$\mathrm{IC}_{50}$} \\
\hline & \multicolumn{5}{|c|}{$\%$ scavenging } & \\
\hline & $20 \mu \mathrm{M}$ & $40 \mu \mathrm{M}$ & $60 \mu \mathrm{M}$ & $80 \mu \mathrm{M}$ & $100 \mu \mathrm{M}$ & \\
\hline D1 & 40.58 & 52.64 & 60.95 & 69.54 & 80.21 & 37.57 \\
\hline D2 & 35.71 & 47.62 & 55.98 & 69.21 & 78.45 & 46.18 \\
\hline D3 & 22.95 & 34.58 & 46.85 & 60.07 & 67.74 & 66.19 \\
\hline D4 & 30.58 & 38.21 & 50.13 & 58.21 & 67.54 & 62.27 \\
\hline D6 & 42.58 & 50.94 & 62.87 & 70.18 & 77.28 & 35.69 \\
\hline D7 & 39.13 & 42.84 & 52.94 & 60.13 & 68.49 & 52.87 \\
\hline D8 & 33.84 & 40.26 & 48.54 & 56.87 & 67.65 & 61.34 \\
\hline D9 & 46.24 & 55.19 & 64.32 & 83.48 & 91.24 & 29.40 \\
\hline D10 & 28.57 & 35.48 & 47.69 & 55.87 & 64.88 & 67.53 \\
\hline D11 & 31.57 & 40.98 & 52.16 & 64.81 & 75.26 & 54.68 \\
\hline D12 & 44.96 & 58.32 & 67.19 & 77.46 & 85.21 & 26.62 \\
\hline D13 & 28.54 & 42.67 & 58.13 & 70.54 & 84.76 & 50.12 \\
\hline D15 & 46.34 & 58.17 & 70.98 & 81.44 & 90.16 & 24.98 \\
\hline D16 & 43.59 & 54.63 & 66.98 & 77.25 & 85.49 & 30.67 \\
\hline D17 & 45.17 & 54.09 & 64.83 & 75.54 & 81.60 & 29.78 \\
\hline D18 & 47.58 & 59.21 & 67.99 & 76.84 & 82.53 & 21.54 \\
\hline D19 & 40.51 & 53.82 & 65.20 & 85.99 & 94.26 & 34.28 \\
\hline D20 & 49.21 & 62.87 & 75.49 & 85.44 & 91.63 & 17.30 \\
\hline D21 & 50.71 & 59.21 & 69.45 & 78.68 & 85.44 & 17.94 \\
\hline D22 & 48.97 & 55.46 & 65.82 & 73.54 & 81.24 & 23.67 \\
\hline D23 & 52.47 & 66.29 & 75.82 & 85.21 & 91.62 & 10.04 \\
\hline D24 & 47.54 & 61.87 & 74.58 & 87.25 & 93.55 & 26.88 \\
\hline D25 & 49.08 & 64.97 & 72.84 & 81.15 & 89.55 & 15.68 \\
\hline D26 & 50.87 & 64.22 & 76.97 & 86.68 & 92.45 & 14.11 \\
\hline D27 & 41.27 & 50.23 & 57.42 & 65.88 & 72.97 & 40.88 \\
\hline D28 & 31.48 & 38.52 & 46.13 & 56.28 & 64.75 & 66.09 \\
\hline D29 & 30.87 & 41.68 & 50.98 & 62.49 & 75.64 & 55.86 \\
\hline D30 & 44.15 & 53.97 & 63.25 & 71.11 & 80.43 & 31.94 \\
\hline Standard & 51.95 & 65.18 & 74.11 & 84.97 & 89.24 & 11.06 \\
\hline
\end{tabular}

$\mathrm{IC}_{50}$ at $10.04 \mu \mathrm{M}$ as compared to standard. The reason may be due to the presence of methoxy group, adjacent to the phenolic hydroxyl group making the molecule as sterically hindered phenol. This observation is in confirmation with the literature that steric hindrance of the phenolic moiety is one of the factors governing antioxidant efficiency [19]. The presence of more phenolic hydroxyl group resulted in an increase of antioxidant activity and indicated that the phenolic moiety is necessary to confer better antioxidant and radical scavenging properties.

\section{In vitro antibacterial activity}

The synthesized 3-hydroxy chromones were evaluated for their in vitro antibacterial activity measured using the agar cup method against two Gram-negative cultures, namely, E. coli, Salmonella typhi and two Grampositive cultures, namely, Staphylococcus aureus, Bacillus subtilis. The activity data are presented in Table 3.

The study revealed that the compounds with a phenolic hydroxyl group exhibited greater antibacterial activity in both Gram-positive and Gramnegative bacteria. Among these, the compound 3-hydroxy-2-(3-hydroxy4-methoxyphenyl)-chromone (D23) showed potent antibacterial activity against all the four strains of bacteria. The compound 3,7-dihydroxy-2-(4-methylphenyl)-chromone (D12) exhibited greater antibacterial activity against Salmonella typhi as compared to standard with a zone of inhibition of $25 \mathrm{~mm}$. The compounds 2-(3-chlorophenyl)3,7-dihydroxy-chromone (D9), 3,7-dihydroxy-2-(4-methylphenyl)chromone (D12), 3,7-dihydroxy-2-(3-nitrophenyl)-4H-1-benzo pyran-4-one (D14), 2-(4-bromophenyl)-3,7-dihydroxy-chromone (D17), 3-hydroxy-2-(3-hydroxy-4-methoxyphenyl)-chromone (D23) and 3,7-dihydroxy-2-(4-methoxyphenyl)-chromone (D26) showed maximum activity against $E$. coli as compared to standard with zone of inhibition of $16 \mathrm{~mm}, 17 \mathrm{~mm}, 12 \mathrm{~mm}, 15 \mathrm{~mm}, 20 \mathrm{~mm}$ and $13 \mathrm{~mm}$, respectively. Thus the main structural feature responsible for antibacterial activity is the hydroxyl group and the 4-oxo group. The weaker antibacterial activity of compounds against gram positive bacteria may be due to the presence of 2,3-double bond as mentioned in the literature [20]. The presence of more phenolic hydroxyl group resulted in an increase of antibacterial activity and indicated that the phenolic moiety is necessary to confer better antibacterial properties.

\section{In vitro antifungal activity}

The synthesized 3-hydroxy chromones were evaluated for their in vitro antifungal activity measured by the Poison plate method against A. niger, $P$. chrysogenum, F. moneliforme and A. flavus. The activity data are presented in Table 4.

The study revealed that almost all the synthesized compounds exhibited greater antifungal activity in all four strains of fungi. Among these, the compound 3-hydroxy-2-(3-hydroxy-4-methoxyphenyl)chromone (D23), 2-(2-chlorophenyl)-3,7-dihydroxy-chromone (D25), 3,7-dihydroxy-2-(4-methoxyphenyl)-chromone (D26) and 2-(furan-2-yl)-3,7-dihydroxy-chromone (D27) showed more than $90 \%$ reduction in growth in all the four strains. Thus the presence of phenolic hydroxyl group and 4-oxo group is also responsible for antifungal activity of title compounds.

\section{CONCLUSION}

An eco-friendly and easy method has been used to synthesize the title compounds. The method includes mild reaction conditions, use of recyclable solvent and easy work-up procedures for the isolation of products. The reaction led to the expected products with high yield and in almost all cases the products obtained in pure form.

The present research work revealed that the compounds of 3-hydroxy chromones containing 2,3-double bond, phenolic substitution and 
Table 3: In vitro antibacterial activity of 3-hydroxy chromones

\begin{tabular}{|c|c|c|c|c|}
\hline \multirow[t]{2}{*}{ Compound } & \multicolumn{4}{|c|}{ Zone of Inhibition (mm) } \\
\hline & Escherichia coli & Salmonella typhi & Staphylococcus aureus & Bacillus subtilis \\
\hline D1 & -ve & - ve & 11 & 17 \\
\hline D3 & -ve & 12 & 16 & 16 \\
\hline D4 & -ve & 12 & 17 & 17 \\
\hline D5 & -ve & -ve & 12 & 12 \\
\hline D6 & -ve & -ve & 18 & 20 \\
\hline D7 & -ve & -ve & 11 & 11 \\
\hline D8 & -ve & -ve & 20 & 20 \\
\hline D9 & 16 & -ve & 22 & 26 \\
\hline D10 & -ve & 12 & 15 & 16 \\
\hline D11 & $-\mathrm{ve}$ & -ve & 11 & 11 \\
\hline D12 & 17 & 25 & 23 & 15 \\
\hline D13 & -ve & -ve & 18 & 17 \\
\hline D14 & 12 & -ve & 21 & 23 \\
\hline D15 & -ve & -ve & 22 & 18 \\
\hline D17 & 15 & -ve & 20 & 25 \\
\hline D18 & -ve & -ve & 18 & 21 \\
\hline D19 & -ve & -ve & 16 & 20 \\
\hline D20 & -ve & -ve & 19 & 20 \\
\hline D21 & -ve & -ve & 20 & 17 \\
\hline D22 & -ve & -ve & 20 & 23 \\
\hline D23 & 20 & 19 & 23 & 25 \\
\hline D24 & -ve & - ve & 20 & 20 \\
\hline D25 & -ve & -ve & 22 & 19 \\
\hline D26 & 13 & -ve & 12 & - ve \\
\hline D27 & -ve & -ve & 20 & 21 \\
\hline D28 & -ve & -ve & 12 & 15 \\
\hline D29 & -ve & -ve & 14 & 14 \\
\hline D30 & -ve & -ve & -ve & 20 \\
\hline DMSO & -ve & -ve & -ve & $-\mathrm{ve}$ \\
\hline Penicillin & 11 & 24 & 36 & 30 \\
\hline
\end{tabular}

Table 4: In vitro antifungal activity of 3-hydroxy chromones

\begin{tabular}{|c|c|c|c|c|}
\hline Compound & Aspergillus niger & Penicillium chrysogenum & Fusarium moneliforme & Aspergillus flavus \\
\hline D1 & RG & RG & RG & RG \\
\hline $\mathrm{D} 2$ & -ve & $\mathrm{RG}$ & -ve & RG \\
\hline D3 & RG & RG & RG & RG \\
\hline D4 & RG & $\mathrm{RG}$ & $\mathrm{RG}$ & RG \\
\hline D5 & RG & $\mathrm{RG}$ & RG & RG \\
\hline D6 & RG & -ve & -ve & RG \\
\hline D7 & RG & RG & RG & RG \\
\hline D9 & RG & $\mathrm{RG}$ & -ve & RG \\
\hline D10 & $\mathrm{RG}$ & -ve & -ve & RG \\
\hline D11 & RG & -ve & -ve & RG \\
\hline D12 & RG & RG & RG & RG \\
\hline D13 & $\mathrm{RG}$ & -ve & -ve & RG \\
\hline D14 & $\mathrm{RG}$ & RG & $\mathrm{RG}$ & RG \\
\hline D15 & $\mathrm{RG}$ & -ve & -ve & $\mathrm{RG}$ \\
\hline D16 & $\mathrm{RG}$ & $\mathrm{RG}$ & $\mathrm{RG}$ & 12 \\
\hline D18 & $\mathrm{RG}$ & -ve & -ve & RG \\
\hline D19 & $\mathrm{RG}$ & -ve & -ve & $\mathrm{RG}$ \\
\hline D20 & $\mathrm{RG}$ & RG & $\mathrm{RG}$ & RG \\
\hline $\mathrm{D} 21$ & -ve & -ve & -ve & -ve \\
\hline D22 & $\mathrm{RG}$ & $\mathrm{RG}$ & $\mathrm{RG}$ & $\mathrm{RG}$ \\
\hline D23 & -ve & -ve & -ve & -ve \\
\hline D24 & RG & -ve & -ve & $\mathrm{RG}$ \\
\hline D25 & -ve & -ve & -ve & -ve \\
\hline D26 & -ve & -ve & -ve & -ve \\
\hline D27 & -ve & --ve & -ve & -ve \\
\hline D28 & -ve & -ve & -ve & $\mathrm{RG}$ \\
\hline D29 & $\mathrm{RG}$ & -ve & -ve & -ve \\
\hline D30 & $\mathrm{RG}$ & -ve & -ve & RG \\
\hline DMSO & +ve & +ve & +ve & +ve \\
\hline Greseofulvin & -ve & -ve & -ve & -ve \\
\hline
\end{tabular}

+ve: Growth (antifungal activity absent), -ve: No growth (more than 90\% reduction in growth); RG: Reduced growth (more than $50 \%$ and $<90 \%$ reduction in growth) 
$\alpha, \beta$-unsaturated keto group showed greater antioxidant activity in the DPPH radical scavenging model. These active compounds also exhibited statistically significant antibacterial and antifungal activity. Hence, these compounds can be developed as useful therapeutic agents after establishing their safe pharmacology and toxicity profile. Nevertheless, the obtained results in all these assays are advocating in terms that additional synthesis of new derivatives and further investigations in this therapeutic area might provide interesting and potentially promising results that can finally be applied for enriching our knowledge and experience in the development of new chemical leads with this specific biological activity.

\section{ACKNOWLEDGMENT}

The authors are very thankful to SAIF, IITM Chennai for providing NMR and Mass spectral data of synthesized compounds.

\section{AUTHORS CONTRIBUTION}

Pallavi Kamble and Sailesh Wadher conceived of the presented idea. Pallavi Kamble carried out the experiment, synthesis and antimicrobial activity of target compounds. Sailesh Wadher encouraged Pallavi Kamble to investigate and supervised the findings of this work. Both authors discussed the results and contributed to the final manuscript. Pallavi Kamble and Sailesh Wadher wrote the manuscript. Sailesh Wadher supervised the project.

\section{CONFLICT OF INTEREST}

The authors confirm that this article content has no conflict of interest.

\section{REFERENCES}

1. Gaspar A, Matos MJ, Garrido J, Uriarte E, Borges F. Chromone: A valid scaffold in medicinal chemistry. Chem Rev 2014;114:4960-92.

2. Hanaa AT, Ewies FE, Wageeh SE. Synthesis of chromones and their applications during the last ten years. Int J Res Pharm Chem 2014;4:1046-85.

3. Edwards AM, Howell JB. The chromones: History, chemistry and clinical development. A tribute to the work of dr R. E. C. Altounyan. Clin Exp Allergy 2000;30:756-74.

4. Dilipkumar P, Preeti V. Flavonoids: A powerful and abundant source of antioxidants. Int J Pharm Pharm Sci 2013;5:95-8.

5. Ju W, Yang ZY, Yi XY, Wang BD. DNA-binding properties studies and spectra of a novel fluorescent zn(ii) complex with a new chromone derivative. Photochem Photobiol 2009;20:183-90.

6. Dengle RV, Deshmukh RN. Synthesis and antimicrobial evaluation of chromones bearing 1, 5-benzothiazepinyl moiety. Int J Pharm Sci Res 2013;4:1495-8.

7. Kajal LJ, Choudhury PK, Maya S. Total flavonoid quantification and to study antibacterial potency of extracts of Butea monosperma flowers, Nigella sativa seeds and Vitex agnus castus leaves. Int J Curr Pharm Res 2017;9:71-4.

8. Inaba T, Tanaka K, Takeno R, Nagaki H, Yoshida C, Takano S, et al. Synthesis and antiinflammatory activity of 7-methanesulfonylamino6-phenoxychromones. Antiarthritic effect of the 3-formylamino compound (T-614) in chronic inflammatory disease models. Chem Pharm Bull (Tokyo) 2000;48:131-9.

9. Judd AS, Souers AJ, Wodka D, Zhao G, Mulhern MM, Iyengar RR, et al. Identification of diamino chromone-2-carboxamides as $\mathrm{MCHr} 1$ antagonists with minimal hERG channel activity. Bioorg Med Chem Lett 2007; 17:2365-71.

10. Durga M, Nathiya S, Devasena T. Immunomodulatory and antioxidant actions of dietary flavonoids. Int J Pharm Pharm Sci 2014;6:50-6.

11. Chengxiang Z, Anton VD, Richard CL. Diversity-oriented synthesis of 3-iodochromones and heteroatom analogues via ICl-induced cyclization. J Org Chem 2006;7:1626-32.

12. Lozinskii OA, Shokol TV, Khilya VP. Synthesis and biological activity of chromones annelated at the $\mathrm{C}(7)-\mathrm{C}(8)$ bond with heterocycles. Chem Heterocycl Compd 2011;47:1055-77.

13. Jayapal MR, Sreenivasa KP, Sreedhar NY. Synthesis and characterization of 2,6-dihydroxy substituted chalcones using peg-400 as a recyclable solvent. J Pharm Sci Res 2010;2:450-8.

14. Conti C, Mastromarino P, Goldoni P, Portalone G, Desideri N. Synthesis and anti-rhinovirus properties of fluoro-substituted flavonoids. Antivir Chem Chemother 2005; 16:267-76.

15. Venkatachalam H, Yogendra N, Jayashree BS. Evaluation of the antioxidant activity of novel synthetic chalcones and flavonols. Int $\mathbf{J}$ Chem Eng Appl 2012;3:216-9.

16. Yogesh M, Ashish G, Pradeep M. Synthesis and antioxidant activity of some chalcones and flavanoids. Int J PharmTech Res 2013;5:811-8.

17. Cruickshank RJ, Duguid P, Swain RR. Medical Microbiology. Vol. 1. New York: Churchill Livingstone Publishers; 1998.

18. Rice-Evans CA, Miller NJ, Paganga G. Structure-antioxidant activity relationships of flavonoids and phenolic acids. Free Radic Biol Med 1996;20:933-56.

19. Pospisil J. Antioxidants: Hindered Phenols. Netherlands: Springer; 1998.

20. Xie Y, Yang W, T ang F, Chen X, Ren L. Antibacterial activities of flavonoids: Structure-activity relationship and mechanism. Curr Med Chem 2015;22:132-49. 